\title{
Nachdenken über Suizidhilfe
}

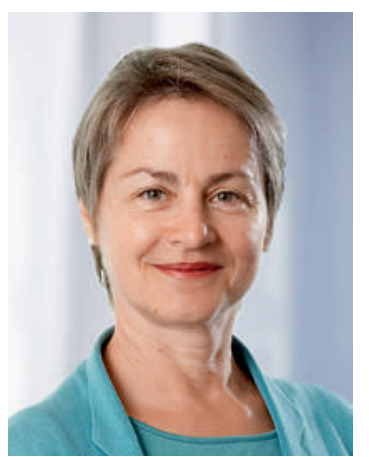

Wer soll, kann, darf oder muss wem wann Suizidhilfe leisten und unter welchen Bedingungen? Diese Fragen beschäftigen uns Ärztinnen und Ärzte seit Jahren, ihre Beantwortung braucht einen gesellschaftlichen Konsens weit über die Medizin hinaus. Wir Ärzte sind hier aber besonders gefordert. Das war nicht immer so: bei der Formulierung von Artikel 115 StGB, der nach wie vor einzigen schweizerischen gesetzlichen Regelung der Sterbehilfe, der diese nur verbietet, wenn sie aus selbstsüchtigen Beweggründen erfolgt, standen nichtgesundheitliche Gründe für einen Suizidwunsch im Vordergrund, und den Ärzten war keine besondere Rolle bei der Durchführung des Suizides zugedacht. Heute hingegen kommt den Ärztinnen und Ärzten eine Schlüsselrolle zu, sind sie es doch, die das Rezept ausstellen für das tödliche Medikament.

Wie gehen Ärztinnen und Ärzte um mit dieser Verantwortung? Wie stehen sie zur Suizidhilfe? Dieser Frage geht eine Studie der SAMW nach, deren Resultate in dieser Ausgabe der Schweizerischen Ärztezeitung auf Seite 1767 dargelegt werden. Den 1318 Ärzten, die sich die Mühe genommen haben, sich mit dem schwierigen Thema differenziert auseinanderzusetzen und den ausführliche Fragebogen zu beantworten, sei hier herzlich gedankt.

Wer über Suizidhilfe nachdenken will, muss unweigerlich zunächst über das Sterben selbst nachdenken, das fällt uns in der Regel schwer. Wer mit Sterbenden zu tun hat, weiss, dass viele Menschen noch kurz vor dem Tod nicht wahrhaben wollen, wie es um sie steht. Und erst recht gilt für gesunde Menschen, dass sie wenig geneigt sind, an den unweigerlich auf sie wartenden eigenen Tod zu denken.

\section{Menschen möchten zunehmend auch selbstbestimmt sterben.}

«O Herr, gib jedem seinen eignen Tod. Das Sterben, das aus jenem Leben geht, darin er Liebe hatte, Sinn und Not», schrieb Rainer Maria Rilke zu Beginn des letzten Jahrhunderts. Auch wenn die Medizin seither bekanntlich grosse Fortschritte gemacht hat, wir länger leben und auch länger gesund bleiben - am Ende erwarten uns Krankheit, Leiden und Tod. Dass wir das Sterben heute bis zu einem gewissen Grad planen können im Sinne von «selbstbestimmt sterben», heisst eigentlich nur, dass wir unsere Lebenszeit verkürzen können, sei es, indem wir bestimmen, welche medizinischen Massnahmen noch durchgeführt werden sollen oder indem wir mit einem assistierten Suizid einen absehbaren Leidensweg verkürzen. Ein paar Jahre länger zu leben oder auch nur ein paar Tage, als es uns eine schwere Krankheit oder die Folgen eines Unfalles oder auch nur das schiere Altern noch zugestehen, das können wir letztlich nicht wählen! Der Tod wird uns gegeben, sagt Rilke.

Diesen begrenzten Spielraum am Lebensende wollen immer mehr Menschen selbst gestalten. So unterschiedlich Menschen gelebt haben, so unterschiedlich wollen sie auch sterben. Eines aber ist allen Menschen gemeinsam: sie bewegen sich in einem Spannungsfeld zwischen dem Wunsch nach Autonomie einerseits und Beziehungswünschen anderer-

\section{Als Ärzte sind wir gefragt, die Autonomie von Patienten in ihrer letzten Lebens- phase zu respektieren, ihnen aber auch den nötigen Rückhalt zu geben.}

seits. Deshalb braucht es nicht nur die Garanten der Autonomie - wie es eine Patientenverfügung zum Beispiel ist -, es braucht ebenso ein Gegenüber, das Anteil nimmt und Fürsorge gewährt - sei das mit guter Palliativmedizin, sei es mit guter Suizidhilfe. Menschen benötigen die Gewissheit, dass Schmerzen gelindert und Ängste ernst genommen werden. Unsere Kultur bewertet Autonomie sehr hoch und nimmt als deren Kehrseite Vereinsamung in Kauf; Abhängigkeit wird gering geschätzt - auf Kosten von Halt und Geborgenheit! Der Sterbeprozess ist wohl die letzte Gelegenheit, die Balance von Autonomie und Abhängigkeit, von Einsamkeit und Geborgenheit noch einmal neu zu tarieren.

So gesehen ist es überaus tröstlich, dass die Haltung der Ärztinnen und Ärzte zu einigen Fragen rund um die Suizidhilfe widersprüchlich ist, wie im Artikel nachzulesen ist. Wenn wir nicht nur die Sterbebegleitung, sondern auch die Suizidhilfe als ein Beziehungsangebot verstehen, muss uns der Tod des Anderen berühren, müssen Ambivalenzen beim Sterbewilligen und bei uns selber wahrgenommen und ausgehalten werden. Nur dann sind wir hilfreiche Begleiter, nur dann können wir die Balance von Autonomie und Abhängigkeit mitgestalten - ein letztes Mal für den Sterbenden und, indem wir mit ihm fühlen, auch für uns selber.

Dr. med. Christine Romann,

Mitglied des Zentralvorstandes der FMH, Departementsverantwortliche Gesundheitsförderung und Prävention 